\title{
Analysis of a Nonautonomous Delayed Predator-Prey System with a Stage Structure for the Predator in a Polluted Environment
}

\author{
G. P. Samanta ${ }^{1,2}$ \\ ${ }^{1}$ Mathematical Institute, Slovak Academy of Sciences, Stefanikova 49, 81473 Bratislava, Slovakia \\ ${ }^{2}$ Department of Mathematics, Bengal Engineering and Science University, Shibpur, Howrah-711103, India \\ Correspondence should be addressed to G. P. Samanta, g_p_samanta@yahoo.co.uk
}

Received 3 July 2009; Accepted 7 February 2010

Academic Editor: Harvinder S. Sidhu

Copyright (C) 2010 G. P. Samanta. This is an open access article distributed under the Creative Commons Attribution License, which permits unrestricted use, distribution, and reproduction in any medium, provided the original work is properly cited.

\begin{abstract}
A two-species nonautonomous Lotka-Volterra type model with diffusional migration among the immature predator population, constant delay among the matured predators, and toxicant effect on the immature predators in a nonprotective patch is proposed. The scale of the protective zone among the immature predator population can be regulated through diffusive coefficients $D_{i}(t)$, $i=1,2$. It is proved that this system is uniformly persistent (permanence) under appropriate conditions. Sufficient conditions are derived to confirm that if this system admits a positive periodic solution, then it is globally asymptotically stable.
\end{abstract}

\section{Introduction}

In recent years, many countries have already realized that the pollution of the environment is a very serious and urgent problem. It is well known that with the rapid development of modern industries and agricultures, a large quantity of toxicants and contaminants enter into ecosystems one after another. One of the most important and meaningful questions in mathematical ecology is the permanence (uniformly persistent) and extinction of a population in a polluted environment. Organisms are often exposed to a polluted environment and take up toxicants. The change in environment is caused by pollution, affecting the long-term survival of species, humans, and biodiversity of the habitat. The question of the effects of pollutants and toxicants on ecological communities is of tremendous important from both environmental and conservational points of view.

Anthropogenically produced toxic substances that enter the aquatic food chain represent a serious health threats to human beings and to the inhabitants of such polluted 
ecosystems. The ability of an aquatic ecosystem to restore itself to the prepollution state is directly related to the compartmental retention times and exchange rates among compartments and the transport rates out of the system. The effect of a toxic substance can be well understood by measuring its presence throughout an aquatic system and by determining the rates of its transport betweencompartments and storage within compartments. An understanding of the compartmental and total system stability of a toxic substance can be achieved with the use of conceptual models [1-4].

Acid rain results from certain kinds of air pollution that mix with precipitation, such as rain or fog, then falls to earth as an acidic solution and its major components are oxides of sulfur and nitrogen that are mainly the by-products of coal-burning power plants, copper melting, factory, and automobile emissions. These oxides are chemically changed in the atmosphere and return to the earth as rain, snow, fog, or dust. In the United states, the most recognized form of acid rain results from sulfur dioxide emissions, which are converted into sulfuric acid in the atmosphere. When this is mixed with precipitation and falls to earth, the effect is precisely like pouring a diluted acid solution onto everything it touches. In lakes also, this acidification process can change ecological structures. In this way toxic substances are invaded into the ecological communities $[5,6]$.

Other examples are oil pollution in the seas [7], degradation of forests [8,9], and dumping of toxic waste in rivers and lakes $[10,11]$.

In order to protect environment and regulate the release of toxic substances wisely, we must assess the harm of the toxicant to the species exposed to it. By using mathematical models, Hallam and Clark [12], Hallam et al. [13, 14], Hallam and De Luna [15], De Luna and Hallam [16], Zhien et al. [17], Huaping and Zhien [18], Freedman and Shukla [19], Wang and Ma [20], Dubey [21], Xiao and Chen [22], Ghosh et al. [23], Buonomo and Lacitignola [24], Li et al. [25], Samanta and Maiti [26], J. Wang and K. Wang [27], He and Wang [28, 29], Das et al. [30], and many others studied the effects of toxic substances on various ecosystems.

The effect of "stage-structure" to the dynamics of predator-prey system is a natural phenomenon and represents the division of a population into immature and mature individuals. As is common, the dynamics-eating habitats, susceptibility to toxicants, and so forth. are often quite different in these two subpopulations. Hence investigation of the effects of such a subdivision on the interaction of species is very much important from the ecological point of view. Stage-structured models have already received much attention by many authors [31, 32]. Many models in mathematical ecology can be formulated as system of differential equations with time delays. The effect of the past history on the stability of system is also an important problem in population ecology. Recently uniformly persistent and stability of a population dynamical system involving time delays have been considered by many authors [33]. Dispersal is a ubiquitous phenomenon in the natural world. Its importance in understanding the ecological and evolutionary dynamics of populations is mirrored by a large number of mathematical models [34]. In order to prevent the destruction of biological resources and to protect the environment, all kinds of measures have been proposed. Establishing a protective patch in mathematical ecology is applied widely. The practical effects of the protective patch on the polluted population is worth investigation.

As species do not exist alone in nature, it is of more biological significance to study the uniformly persistent-extinction threshold of each population in systems of two or more interacting species subject to organismal and environmental toxicants. In most of the classical toxicant-population models it is assumed that each individual has the same dose-response parameter to the organismal toxicant concentration regardless of the difference between the mature and immature populations. However, in the natural world, there are many species 
whose individual members have a life history that takes them through two stages, immature and mature. Moreover, there is a big difference between the immature and mature species in many aspects. For example, for less concentration of $\mathrm{C}_{2} \mathrm{H}_{4}$ in an environment, the immature cymbidium is so susceptible that it begins to wither, whereas, the mature cymbidium often has not been affected $[22,35]$. Therefore, it is important to consider the effect of stage structure on population growth in a polluted environment. In this paper, we have considered pollution in a nonautonomous predator-prey system together with diffusional migration among the immature predator population between protective and nonprotective patches where there is a constant delay among the matured predators due to the gestation of the predator based on the assumption that mature adult predators can only contribute to the reproduction of the predator biomass and that the change rate of predators depends on the number of the preys and of the predators presented at some previous time. The scale of the protective zone among the immature predator population can be regulated through diffusive coefficients $D_{i}(t), i=$ 1,2. Here we incorporate stage-structure for the predator and assume that the toxicants have no effect on the mature species. This seems to be reasonable. The main feature of the present paper is to study the asymptotical behaviour of the toxicant-population model with stage structure, diffusional migration, and time delay so as to obtain some conditions under which the population is uniformly persistent. In addition, by constructing an appropriate Lyapunov function, we have derived sufficient conditions to confirm that if the system admits a positive periodic solution, then it is globally asymptotically stable.

\section{The Basic Mathematical Model}

Our study is based on the hypothesis of a complete spatial homogeneous environment. We incorporate stage-structure for the predator into a nonautonomous Lotka-Volterra type predator-prey model. We consider the following delayed model to describe the dynamics of prey and predator population in a polluted environment. The state variables of the model are $x(t), y_{1}(t), y_{2}(t)$, and $y_{3}(t)$, the densities of the prey, immature predator in nonprotective patch (with toxicants), immature predator in protective patch (without toxicants), and mature predator species at time $t$, respectively, $C_{0}(t)$ is the concentration of toxicant in the immature predator organism in nonprotective patch (with toxicants) at time $t$, and $C_{e}(t)$ is the concentration of toxicant in the environment at time $t$. Here we assume that the toxicants have no effect on the mature species and immature predator in protective patch.

Let us consider the two-species nonautonomous Lotka-Volterra type model with diffusional migration among the immature predator population, constant delay among the matured predators, and toxicant effect on immature predators in nonprotective patch:

$$
\begin{aligned}
& \frac{d x(t)}{d t}=x(t)\left[r_{1}(t)-a_{11}(t) x(t)-a_{12}(t) y_{3}(t)\right], \\
& \frac{d y_{1}(t)}{d t}=b_{1}(t) y_{3}(t)-\theta_{1}(t) y_{1}(t)-\delta_{1}(t) y_{1}(t)-d(t) C_{0}(t) y_{1}(t)-\beta_{1}(t) y_{1}^{2}(t)+D_{1}(t)\left(y_{2}(t)-y_{1}(t)\right), \\
& \frac{d y_{2}(t)}{d t}=b_{2}(t) y_{3}(t)-\theta_{2}(t) y_{2}(t)-\delta_{2}(t) y_{2}(t)-\beta_{2}(t) y_{2}^{2}(t)+D_{2}(t)\left(y_{1}(t)-y_{2}(t)\right),
\end{aligned}
$$




$$
\begin{aligned}
& \frac{d y_{3}(t)}{d t}=y_{3}(t)\left[-r_{2}(t)-a_{22}(t) y_{3}(t)\right]+a_{21}(t) y_{3}(t-\tau) x(t-\tau)+\theta_{1}(t) y_{1}(t)+\theta_{2}(t) y_{2}(t), \\
& \frac{d C_{0}(t)}{d t}=k(t) C_{e}(t)+l(t)-g(t) C_{0}(t)-m(t) C_{0}(t), \\
& \frac{d C_{e}(t)}{d t}=-h(t) C_{e}(t)+u(t) .
\end{aligned}
$$

The model is derived under following assumptions.

(A1) The prey population: the growth of the species is of a Lotka-Volterra nature. At any time $t>0$, the birth rate is proportional to the existing prey population with proportional function $r_{1}(t)$ and $a_{11}(t)$ is the intraspecific competition rate function of the prey. $r_{1}(t)$ and $a_{11}(t)$ are continuous, bounded, and strictly positive functions in the interval $[0, \infty)$.

(A2) The predator population: the predators cannot hunt prey when the predators are infant (immature). The mature predators feed only on prey; $a_{12}(t)$ is the capturing rate function of the predator, $a_{21}(\mathrm{t}) / a_{12}(t)$ is the conversion rate function of nutrients into the reproduction of the mature predator, and $\tau \geq 0$ is a constant delay due to the gestation of the mature predator based on the assumption that mature adult predators can only contribute to the reproduction of the predator biomass and that the change rate of predators depends on the number of the preys and of the predators presented at some previous time. To protect the immature predator population, the region $(\Psi)$ of immature predator population is divided into two patches $\Psi_{1}$ and $\Psi_{2}$. Pollution is permitted in $\Psi_{1}$ and is inhibited in $\Psi_{2}$. We call $\Psi_{1}$ and $\Psi_{2}$ as the nonprotective and protective patches, respectively. Since the difference of densities between patches $\Psi_{1}$ and $\Psi_{2}$ exists, the diffusive migration can occur between these two patches, and $D_{1}(t), D_{2}(t)$ are diffusion coefficient functions of nonprotective immature and protective immature predator species, respectively. At any time $t>0$, the rates of transitions from nonprotective immature and protective immature individuals to mature individuals are proportional to the existing nonprotective immature and protective immature populations, respectively, with proportional functions $\theta_{1}(t)$ and $\theta_{2}(t)$, respectively, and the death rates of mature, nonprotective immature, and protective immature population are proportional to the existing mature, nonprotective immature, and protective immature population, respectively, with proportional functions $r_{2}(t), \delta_{1}(t)$, and $\delta_{2}(t)$, respectively. The birth rates of nonprotective immature and protective immature population are proportional to the existing mature population with proportional functions $b_{1}(t)$ and $b_{2}(t)$, respectively. $a_{22}(t), \beta_{1}(t)$, and $\beta_{2}(t)$ are the intraspecific competition rate functions of the mature, nonprotective immature, and protective immature population, respectively. $a_{12}(t), a_{21}(t), a_{22}(t), D_{i}(t), \theta_{i}(t), r_{2}(t), \delta_{i}(t), b_{i}(t)$, and $\beta_{i}(t),(i=$ $1,2)$ are continuous, bounded, and strictly positive functions in the interval $[0, \infty)$.

(A3) Toxicants: $d(t)$ is the dose response parameter of nonprotective immature predator species $y_{1}(t)$ to the organismal toxicant concentration. The first two terms on the right of the fifth equation in system (2.1) denote the organismal net uptake of toxicant from the environment $\left(k(t) C_{e}(t)\right)$ and the food chain $(l(t))$, respectively, 
and the third and fourth terms represent the organismal net loss of toxicant due to metabolic processing and other causes. $k(t)$ denotes environmental toxicant uptake rate per unit mass organism, $l(t)$ denotes uptake rate of toxicant in food per unit mass organism, and $-g(t) C_{o}(t)$ and $-m(t) C_{o}(t)$ represent the egestion and depuration rates of the toxicant in the organism, respectively. The first term $\left(-h(t) C_{e}(t)\right)$ on the right of the sixth equation in system (2.1) represents the toxicant loss from the environment itself by biological transformation, chemical hydrolysis, volatilization, microbial degradation, photosynthetic degradation, and so on and the exogenous rate of input of toxicant into the environment is represented by $u(t)$. The capacity of the environment is so large that the change of toxicants in the environment that comes from uptake and egestion by the organisms can be neglected [15, 36, 37]. The coefficients $d(t), k(t), l(t), g(t), m(t), h(t)$, and $u(t)$ are assumed to be nonnegative, continuous, and bounded functions in the interval $[0, \infty)$.

The initial conditions for system (2.1) take the form

$$
\begin{gathered}
x(\theta)=\phi_{1}(\theta), \quad y_{i}(\theta)=\psi_{i}(\theta), \quad C_{0}(\theta)=\phi_{2}(\theta), \quad C_{e}(\theta)=\phi_{3}(\theta), \quad(i=1,2,3), \\
\phi_{i}(\theta) \geq 0, \quad \psi_{i}(\theta) \geq 0 \quad(i=1,2,3), \theta \in[-\tau, 0], \\
x(0)>0, \quad y_{i}(0)>0, \quad 0 \leq C_{0}(0) \leq 1, \quad 0 \leq C_{e}(0) \leq 1 \quad(i=1,2,3),
\end{gathered}
$$

where $\Phi=\left(\phi_{1}(\theta), \phi_{2}(\theta), \phi_{3}(\theta), \psi_{1}(\theta), \psi_{2}(\theta), \psi_{3}(\theta)\right) \in C\left([-\tau, 0], R_{+}^{6}\right)$, the Banach space of continuous functions mapping the interval $[-\tau, 0]$ into $R_{+}^{6}$, where $R_{+}^{6}=\left\{\left(x_{1}, x_{2}, x_{3}, x_{4}, x_{5}, x_{6}\right)\right.$ : $\left.x_{i} \geq 0, i=1,2,3,4,5,6\right\}$.

It is well known by the fundamental theory of functional differential equations [38] that system (2.1) has a unique solution $\left(x(t), y_{1}(t), y_{2}(t), y_{3}(t), C_{0}(t), C_{e}(t)\right)$ satisfying initial conditions (2.2). on $[0,+\infty)$.

Let $f^{l}=\inf _{t \geq 0} f(t), f^{u}=\sup _{t \geq 0} f(t)$, for a continuous and bounded function $f(t)$ defined

In this paper, we always assume that the following holds:

$$
\begin{array}{cl}
\min \left\{d^{l}, k^{l}, l^{l}, g^{l}, m^{l}, h^{l}, u^{l}\right\} \geq 0, & \min _{i, j=1,2}\left\{r_{i}^{l}, a_{i j}^{l}, b_{i}^{l}, \theta_{i}^{l}, \delta_{i}^{l}, \beta_{i}^{l}, D_{i}^{l}\right\}>0, \\
\max \left\{d^{u}, k^{u}, l^{u}, g^{u}, m^{u}, h^{u}, u^{u}\right\}<\infty, & \max _{i, j=1,2}\left\{r_{i}^{u}, a_{i j}^{u}, b_{i}^{u}, \theta_{i}^{u}, \delta_{i}^{u}, \beta_{i}^{u}, D_{i}^{u}\right\}<\infty .
\end{array}
$$

Each of $C_{0}(t)$ and $C_{e}(t)$ is a concentration, and thus, these variables cannot be greater than 1 . So we should give some conditions, such that

$$
0 \leq C_{0}(t) \leq 1, \quad 0 \leq C_{e}(t) \leq 1, \quad \forall t \geq 0 .
$$

Theorem 2.1. Every solution of system (2.1) with initial conditions (2.2) exists and is unique in the interval $[0, \infty)$ and $x(t)>0, y_{1}(t)>0, y_{2}(t)>0, y_{3}(t)>0, C_{0}(t) \geq 0, C_{e}(t) \geq 0$, for all $t \geq 0$.

Proof. Since the right-hand side of system (2.1) is completely continuous and locally Lipschitzian on $C$, the solution $\left(x(t), y_{1}(t), y_{2}(t), y_{3}(t), C_{0}(t), C_{e}(t)\right)$ of $(2.1)$ with initial 
conditions (2.2) exists and is unique on $[0, \alpha)$, where $0<\alpha \leq+\infty$ [38, chapter 2]. From system (2.1) with initial conditions (2.2), we have

$$
x(t)=x(0) \exp \int_{0}^{t}\left[r_{1}(s)-a_{11}(s) x(s)-a_{12}(s) y_{3}(s)\right] d s>0, \quad \forall t \geq 0 .
$$

Next, we show that $y_{1}(t)>0$ for all $t \in[0, \infty)$. Otherwise, there exists a $t_{1} \in(0, \infty)$ such that $y_{1}\left(t_{1}\right)=0, \dot{y}_{1}\left(t_{1}\right) \leq 0$, and $y_{1}(t)>0$ for all $t \in\left[0, t_{1}\right)$. Hence there must have $y_{2}(t)>0$ for all $t \in\left[0, t_{1}\right)$. If this statement is not true, then there exists a $t_{2} \in\left(0, t_{1}\right)$ such that $y_{2}\left(t_{2}\right)=0$ and $y_{2}(t)>0$ on $\left[0, t_{2}\right)$. We claim that $y_{3}(t)>0$ for all $t \in\left[0, t_{2}\right)$. If this statement is not true, then there exists a $t_{3} \in\left(0, t_{2}\right)$ such that $y_{3}\left(t_{3}\right)=0$ and $y_{3}(t) \geq 0$ for all $t \in\left[-\tau, t_{3}\right]$. Furthermore,

$$
\frac{d y_{3}(t)}{d t} \geq-r_{2}(t) y_{3}(t)-a_{22}(t) y_{3}^{2}(t), \quad \forall t \in\left[0, t_{3}\right]
$$

then, $y_{3}(t) \geq y_{3}(0) \exp \int_{0}^{t}\left[-r_{2}(s)-a_{22}(s) y_{3}(s)\right] d s>0$, for all

$$
t \in\left[0, t_{3}\right] \Longrightarrow y_{3}\left(t_{3}\right) \geq y_{3}(0) \exp \int_{0}^{t_{3}}\left[-r_{2}(s)-a_{22}(s) y_{3}(s)\right] d s>0
$$

which is a contradiction. Hence, $y_{3}(t)>0$ for all $t \in\left[0, t_{2}\right)$. Integrating the third equation of system (2.1) from 0 to $t_{2}$, we have

$$
\begin{aligned}
y_{2}\left(t_{2}\right)= & y_{2}(0) \exp \left\{-\int_{0}^{t_{2}}\left(D_{2}(s)+\theta_{2}(s)+\delta_{2}(s)+\beta_{2}(s) y_{2}(s)\right) d s\right\} \\
& +\int_{0}^{t_{2}}\left(b_{2}(u) y_{3}(u)+D_{2}(u) y_{1}(u)\right) \exp \left\{\int_{t_{2}}^{u}\left(D_{2}(s)+\theta_{2}(s)+\delta_{2}(s)+\beta_{2}(s) y_{2}(s)\right) d s\right\} d u>0,
\end{aligned}
$$

which is a contradiction with $y_{2}\left(t_{2}\right)=0$. So $y_{2}(t)>0$, for all $t \in\left[0, t_{1}\right)$ and hence $y_{3}(t)>0$, for all $t \in\left[0, t_{1}\right)$. Integrating the second equation of system (2.1) from 0 to $t_{1}$, we have

$$
\begin{aligned}
& y_{1}\left(t_{1}\right)= y_{1}(0) \exp \left\{-\int_{0}^{t_{1}}\left(D_{1}(s)+\theta_{1}(s)+\delta_{1}(s)+d(s) C_{0}(s)+\beta_{1}(s) y_{1}(s)\right) d s\right\} \\
&+\int_{0}^{t_{1}}\left(b_{1}(u) y_{3}(u)+D_{1}(u) y_{2}(u)\right) \exp \left\{\int _ { t _ { 1 } } ^ { u } \left(D_{1}(s)+\theta_{1}(s)+\delta_{1}(s)\right.\right. \\
&\left.\left.+d(s) C_{0}(s)+\beta_{1}(s) y_{1}(s)\right) d s\right\} d u>0,
\end{aligned}
$$

which is a contradiction with $y_{1}\left(t_{1}\right)=0$. So $y_{1}(t)>0$, for all $t \geq 0$ and hence $y_{2}(t)>0, y_{3}(t)>$ 0 , for all $t \geq 0$. 
Also,

$$
\begin{gathered}
C_{0}(t)=e^{-\int_{0}^{t}\{m(s)+g(s)\} d s}\left[\int_{0}^{t}\left\{k(s) C_{e}(s)+l(s)\right\} e^{\int_{0}^{s}\{m(s)+g(s)\} d s} d s+C_{0}(0)\right] \geq 0, \quad \forall t \geq 0, \\
C_{e}(t)=e^{-\int_{0}^{t} h(s) d s}\left[\int_{0}^{t} u(s) e^{\int_{0}^{s} h(s) d s} d s+C_{e}(0)\right] \geq 0 \quad \forall t \geq 0 .
\end{gathered}
$$

This completes the proof.

Theorem 2.2. From system (2.1) with initial conditions (2.2), if $k^{u}+l^{u} \leq g^{l}+m^{l}, u^{u} \leq h^{l}$, then $0 \leq C_{0}(t) \leq 1,0 \leq C_{e}(t) \leq 1$, for all $t \geq 0$.

Proof. According to Theorem 2.1, we have $C_{0}(t) \geq 0, C_{e}(t) \geq 0$, for all $t \geq 0$. Now we have to prove that $C_{0}(t) \leq 1, C_{e}(t) \leq 1$, for all $t \geq 0$.

If the conclusion is false, then the maximum interval is $[0, T]$ such that $0 \leq C_{0}(t) \leq$ $1,0 \leq C_{e}(t) \leq 1$, for all $t \in[0, T]$ and at least one of the following cases will arise:

(i) $C_{0}(T)=1, C_{e}(T)<1$;

(ii) $C_{0}(T)<1, C_{e}(T)=1$;

(iii) $C_{0}(T)=C_{e}(T)=1$.

We will prove that none of these cases is true.

(i) $C_{0}(T)=1, C_{e}(T)<1$; using the condition $k^{u}+l^{u} \leq g^{l}+m^{l}$, we have

$$
\begin{aligned}
\left.\frac{d C_{0}(t)}{d t}\right|_{t=T} & =k(T) C_{e}(T)+l(T)-g(T) C_{0}(T)-m(T) C_{0}(T) \\
& <k(T)+l(T)-g(T)-m(T) \\
& \leq k^{u}+l^{u}-g^{l}-m^{l} \leq 0
\end{aligned}
$$

thus there exist $t_{1}>0$, s.t. $0 \leq C_{0}(t) \leq 1,0 \leq C_{e}(t) \leq 1$, for all $t \in\left[T, T+t_{1}\right]$. This contradicts the definition of the interval $[0, T]$. So there is no $T$ such that $0 \leq C_{0}(t) \leq 1,0 \leq C_{e}(t) \leq 1$, for all $t \in[0, T]$ with $C_{0}(T)=1, C_{e}(T)<1$.

With the same reasoning as in case (i), for cases (ii) and (iii), as far as $t$ which keeps $0 \leq C_{0}(t) \leq 1$ and $0 \leq C_{e}(t) \leq 1$ is concerned, the interval $[0, T]$ can be extended rightwards. This contradicts the property of $T$. Therefore, $0 \leq C_{0}(t) \leq 1,0 \leq C_{e}(t) \leq 1$, for all $t \geq 0$. This completes the proof. 
Putting the expression of $C_{e}(t)$ in $C_{0}(t)$, we can express $C_{0}(t)$ in terms of some bounded continuous functions; therefore the system (2.1) and (2.2) may be simplified as follows:

$$
\begin{gathered}
\frac{d x(t)}{d t}=x(t)\left[\mathrm{r}_{1}(t)-a_{11}(t) x(t)-a_{12}(t) y_{3}(t)\right] \\
\frac{d y_{1}(t)}{d t}=b_{1}(t) y_{3}(t)-\theta_{1}(t) y_{1}(t)-\delta_{1}(t) y_{1}(t)-d(t) C_{0}(t) y_{1}(t)-\beta_{1}(t) y_{1}^{2}(t)+D_{1}(t)\left(y_{2}(t)-y_{1}(t)\right) \\
\frac{d y_{2}(t)}{d t}=b_{2}(t) y_{3}(t)-\theta_{2}(t) y_{2}(t)-\delta_{2}(t) y_{2}(t)-\beta_{2}(t) y_{2}^{2}(t)+D_{2}(t)\left(y_{1}(t)-y_{2}(t)\right) \\
\frac{d y_{3}(t)}{d t}=y_{3}(t)\left[-r_{2}(t)-a_{22}(t) y_{3}(t)\right]+a_{21}(t) y_{3}(t-\tau) x(t-\tau)+\theta_{1}(t) y_{1}(t)+\theta_{2}(t) y_{2}(t)
\end{gathered}
$$

The initial conditions are

$$
\begin{gathered}
x(\theta)=\phi_{1}(\theta), \quad y_{i}(\theta)=\psi_{i}(\theta) \quad(i=1,2,3), \\
\phi_{1}(\theta) \geq 0, \quad \psi_{i}(\theta) \geq 0 \quad(i=1,2,3), \theta \in[-\tau, 0], \\
x(0)>0, \quad y_{i}(0)>0 \quad(i=1,2,3),
\end{gathered}
$$

where $\Phi=\left(\phi_{1}(\theta), \psi_{1}(\theta), \psi_{2}(\theta), \psi_{3}(\theta)\right) \in C\left([-\tau, 0], R_{+}^{4}\right)$, the Banach space of continuous functions mapping the interval $[-\tau, 0]$ into $R_{+}^{4}$, where $R_{+}^{4}=\left\{\left(x_{1}, x_{2}, x_{3}, x_{4}\right): x_{i} \geq 0, i=\right.$ $1,2,3,4\}$.

\section{Uniformly Persistent of System (2.12)}

Here we wish to discuss the uniformly persistent of the system (2.12) with initial conditions (2.13), which demonstrates how this system will be uniformly persistent, is that, the longterm survival (i.e., will not vanish in time) of all components of the system (2.12) with initial conditions (2.13), under some conditions.

Definition 3.1. The system (2.12) is said to be uniformly persistent, that is, the long-term survival (will not vanish in time) of all components of the system (2.12), if there are positive constants $v_{i}$ and $w_{i} \quad(i=1,2,3,4)$ such that

$$
\begin{aligned}
& v_{1} \leq \liminf _{t \rightarrow \infty} x(t) \leq \limsup _{t \rightarrow \infty} x(t) \leq w_{1}, \\
& v_{2} \leq \liminf _{t \rightarrow \infty} y_{1}(t) \leq \limsup _{t \rightarrow \infty} y_{1}(t) \leq w_{2}, \\
& v_{3} \leq \liminf _{t \rightarrow \infty} y_{2}(t) \leq \limsup _{t \rightarrow \infty} y_{2}(t) \leq w_{3}, \\
& v_{4} \leq \liminf _{t \rightarrow \infty} y_{3}(t) \leq \limsup _{t \rightarrow \infty} y_{3}(t) \leq w_{4}
\end{aligned}
$$


hold for any solution $\left(x(t), y_{1}(t), y_{2}(t), y_{3}(t)\right)$ of (2.12) with initial conditions (2.13). Here $v_{i}$ and $w_{i}(i=1,2,3,4)$ are independent of (2.13).

Theorem 3.2 (see [39]). Consider the following equation:

$$
\dot{x}(t)=a x(t-\tau)-b x(t)-c x^{2}(t),
$$

where $a, b, c, \tau>0 ; x(t)>0$, for $-\tau \leq t \leq 0$. One has the following:

(I) if $a>b$, then $\lim _{t \rightarrow \infty} x(t)=(a-b) / c$;

(II) if $a<b$, then $\lim _{t \rightarrow \infty} x(t)=0$.

Theorem 3.3. Let $X(t)=\left(x(t), y_{1}(t), y_{2}(t), y_{3}(t)\right)^{T}$ denote any solution of system (2.12) and (2.13). Suppose that system (2.12) satisfies $b_{1}^{u}>\theta_{1}^{l}+\delta_{1}^{l}+d^{l} C_{0}^{l}, b_{2}^{u}>\theta_{2}^{l}+\delta_{2}^{l}$, and $a_{21}^{u} r_{1}^{u}>a_{11}^{l}\left(r_{2}^{l}-\theta_{1}^{u}-\theta_{2}^{u}\right)>0$. Then there exist a $T_{3}>0$ such that

$$
\begin{gathered}
x(t) \leq M_{1}, \quad y_{i}(t) \leq M_{4} \quad(i=1,2,3), \quad \forall t \geq T_{3}, \text { where } M_{1}>\frac{r_{1}^{u}}{a_{11}^{l}}, \\
M_{4}>M^{*}=\max \left\{\frac{b_{1}^{u}-\theta_{1}^{l}-\delta_{1}^{l}-d^{l} C_{0}^{l}}{\beta_{1}^{l}}, \frac{b_{2}^{u}-\theta_{2}^{l}-\delta_{2}^{l}}{\beta_{2}^{l}}, \frac{a_{21}^{u} r_{1}^{u}-a_{11}^{l}\left(r_{2}^{l}-\theta_{1}^{u}-\theta_{2}^{u}\right)}{a_{11}^{l} a_{22}^{l}}\right\} .
\end{gathered}
$$

Proof. Let $M_{1}>r_{1}^{u} / a_{11}^{l}$. We have $\dot{x}(t) \leq x(t)\left[r_{1}(t)-a_{11}(t) x(t)\right] \leq x(t)\left[r_{1}^{u}-a_{11}^{l} x(t)\right]$. Therefore, if $x(0) \leq M_{1}$, then $x(t) \leq M_{1}$, for all $t \geq 0$. If $x(0)>M_{1}$, and let $-\alpha_{1}=M_{1}\left(r_{1}^{u}-a_{11}^{l} M_{1}\right), \alpha_{1}>0$, then there exist an $\epsilon_{1}>0$, s.t. if $t \in\left[0, \epsilon_{1}\right), x(t)>M_{1}$, and we have $\dot{x}(t)<-\alpha_{1}<0$. Therefore, there exist a $T_{1}>0$ s.t. $x(t) \leq M_{1}$, for all $t \geq T_{1}$, where $M_{1}>r_{1}^{u} / a_{11}^{l}$. We define $V(t)=$ $\max \left\{y_{1}(t), y_{2}(t), y_{3}(t)\right\}$. Calculating the upper-right derivative of $V(t)$ along the solution of system (2.12) and (2.13), we have the followings:

(1) if $y_{1}(t) \geq y_{2}(t) \geq y_{3}(t)$, or $y_{1}(t) \geq y_{3}(t) \geq y_{2}(t)$, then

$$
\begin{aligned}
D^{+} V(t) & =\dot{y}_{1}(t) \\
& \leq b_{1}^{u} y_{1}(t)-\theta_{1}^{l} y_{1}(t)-\delta_{1}^{l} y_{1}(t)-d^{l} C_{0}^{l} y_{1}(t)-\beta_{1}^{l} y_{1}^{2}(t) \\
& =\left(b_{1}^{u}-\theta_{1}^{l}-\delta_{1}^{l}-d^{l} C_{0}^{l}\right) y_{1}(t)-\beta_{1}^{l} y_{1}^{2}(t), \quad \forall t \geq 0
\end{aligned}
$$

(2) if $y_{2}(t) \geq y_{1}(t) \geq y_{3}(t)$, or $y_{2}(t) \geq y_{3}(t) \geq y_{1}(t)$, then

$$
\begin{aligned}
D^{+} V(t) & =\dot{y}_{2}(t) \\
& \leq b_{2}^{u} y_{2}(t)-\theta_{2}^{l} y_{2}(t)-\delta_{2}^{l} y_{2}(t)-\beta_{2}^{l} y_{2}^{2}(t) \\
& =\left(b_{2}^{u}-\theta_{2}^{l}-\delta_{2}^{l}\right) y_{2}(t)-\beta_{2}^{l} y_{2}^{2}(t), \quad \forall t \geq 0 ;
\end{aligned}
$$


(3) if $y_{3}(t) \geq y_{1}(t) \geq y_{2}(t)$, or, $y_{3}(t) \geq y_{2}(t) \geq y_{1}(t)$, then

$$
\begin{aligned}
D^{+} V(t) & =\dot{y}_{3}(t) \\
& \leq-r_{2}^{l} y_{3}(t)-a_{22}^{l} y_{3}^{2}(t)+a_{21}^{u} M_{1} y_{3}(t-\tau)+\left(\theta_{1}^{u}+\theta_{2}^{u}\right) y_{3}(t) \\
& =-\left(r_{2}^{l}-\theta_{1}^{u}-\theta_{2}^{u}\right) y_{3}(t)-a_{22}^{l} y_{3}^{2}(t)+a_{21}^{u} M_{1} y_{3}(t-\tau), \quad \forall t \geq T_{1}+\tau .
\end{aligned}
$$

Let

$$
M_{2}>M_{1}^{*}=\max \left\{\frac{b_{1}^{u}-\theta_{1}^{l}-\delta_{1}^{l}-d^{l} C_{0}^{l}}{\beta_{1}^{l}}, \frac{b_{2}^{u}-\theta_{2}^{l}-\delta_{2}^{l}}{\beta_{2}^{l}}\right\} .
$$

From (3.4) and (3.5), we can obtain the followings:

(i) if $\max \left\{y_{1}(0), y_{2}(0), y_{3}(0)\right\} \leq M_{2}$, then $\max \left\{y_{1}(t), y_{2}(t), y_{t}(t)\right\} \leq M_{2}$, for all $t \geq 0$;

(ii) if $\max \left\{y_{1}(0), y_{2}(0), y_{3}(0)\right\}>M_{2}$, and let $-\alpha_{2}=\max \left[M_{2}\left\{\left(b_{1}^{u}-\theta_{1}^{l}-\delta_{1}^{l}-d^{l} C_{0}^{l}\right)-\right.\right.$ $\left.\left.\beta_{1}^{l} M_{2}\right\}, M_{2}\left\{\left(b_{2}^{u}-\theta_{2}^{l}-\delta_{2}^{l}\right)-\beta_{2}^{l} M_{2}\right\}\right], \alpha_{2}>0$, we consider the following two cases:

(a) $V(0)=y_{1}(0)>M_{2}$,

(b) $V(0)=y_{2}(0)>M_{2}$.

If (a) holds, then there exist an $\epsilon_{2}>0$, s.t. if $t \in\left[0, \epsilon_{2}\right), V(t)=y_{1}(t)>M_{2}$, and we have $D^{+} V(t)=\dot{y}_{1}(t)<-\alpha_{2}<0$.

If (b) holds, then there exist an $\epsilon_{3}>0$, s.t. if $t \in\left[0, \epsilon_{3}\right), V(t)=y_{2}(t)>M_{2}$, and we have $D^{+} V(t)=\dot{y}_{2}(t)<-\alpha_{2}<0$.

If (3.6) holds, then by Theorem 3.2, there exist $T_{2} \geq T_{1}+\tau>0$, s.t.

$$
\begin{gathered}
y_{3}(t)<M_{3}, \quad \forall t \geq T_{2} \geq T_{1}+\tau>0, \text { where } M_{3}>\frac{a_{21}^{u} r_{1}^{u}-a_{11}^{l}\left(r_{2}^{l}-\theta_{1}^{u}-\theta_{2}^{u}\right)}{a_{11}^{l} a_{22}^{l}} \\
\left(\text { since } M_{1} \text { can be chosen close enough to } \frac{r_{1}^{u}}{a_{11}^{l}}\right) .
\end{gathered}
$$

From the above discussions, we conclude that there exist $T_{3} \geq T_{2} \geq T_{1}+\tau>0$, s.t.

$$
\begin{gathered}
x(t) \leq M_{1}, \quad y_{i}(t) \leq M_{4} \quad(i=1,2,3), \quad \forall t \geq T_{3}, \quad \text { where } M_{1}>\frac{r_{1}^{u}}{a_{11}^{l}}, \\
M_{4}>M^{*}=\max \left\{\frac{b_{1}^{u}-\theta_{1}^{l}-\delta_{1}^{l}-d^{l} C_{0}^{l}}{\beta_{1}^{l}}, \frac{b_{2}^{u}-\theta_{2}^{l}-\delta_{2}^{l}}{\beta_{2}^{l}}, \frac{a_{21}^{u} r_{1}^{u}-a_{11}^{l}\left(r_{2}^{l}-\theta_{1}^{u}-\theta_{2}^{u}\right)}{a_{11}^{l} a_{22}^{l}}\right\} .
\end{gathered}
$$

This completes the proof. 
Theorem 3.4. Suppose that system (2.12) and (2.13) satisfies the following conditions:

$$
\begin{aligned}
b_{1}^{l}>\theta_{1}^{u}+\delta_{1}^{u}+ & d^{u} C_{0}^{u}, \quad b_{2}^{l}>\theta_{2}^{u}+\delta_{2}^{u}, \quad r_{2}^{l}>\theta_{1}^{u}+\theta_{2}^{u}, \quad a_{21}^{l} r_{1}^{l}>a_{11}^{u}\left(r_{2}^{u}-\theta_{1}^{l}-\theta_{2}^{l}\right) \\
\max & \left\{\frac{b_{1}^{u}-\theta_{1}^{l}-\delta_{1}^{l}-d^{l} C_{0}^{l}}{\beta_{1}^{l}}, \frac{b_{2}^{u}-\theta_{2}^{l}-\delta_{2}^{l}}{\beta_{2}^{l}}, \frac{a_{21}^{u} r_{1}^{u}-a_{11}^{l}\left(r_{2}^{l}-\theta_{1}^{u}-\theta_{2}^{u}\right)}{a_{11}^{l} a_{22}^{l}}\right\} \\
& <M_{4}<\min \left\{\frac{r_{1}^{l}}{a_{12}^{u}}, \frac{a_{21}^{l} r_{1}^{l}-a_{11}^{u}\left(r_{2}^{u}-\theta_{1}^{l}-\theta_{2}^{l}\right)}{a_{21}^{l} a_{12}^{u}}\right\} .
\end{aligned}
$$

Then system (2.12) and (2.13) is uniformly persistent.

Proof. Let $X(t)=\left(x(t), y_{1}(t), y_{2}(t), y_{2}(t)\right)^{T}$ be a solution of (2.12) and (2.13). $\therefore \dot{x}(t) \geq x(t)\left[r_{1}^{l}-\right.$ $\left.a_{11}^{u} x(t)-a_{12}^{u} M_{4}\right]$, for all $t \geq T_{3}$ (using Theorem 3.3 and $T_{3}$ is defined there). Now, $M_{4}<$ $\left(a_{21}^{l} r_{1}^{l}-a_{11}^{u}\left(r_{2}^{u}-\theta_{1}^{l}-\theta_{2}^{l}\right)\right) / a_{21}^{l} a_{12}^{u}$, and $r_{2}^{l}>\theta_{1}^{u}+\theta_{2}^{u}$ together imply $0<\left(r_{2}^{u}-\theta_{1}^{l}-\theta_{2}^{l}\right) / a_{21}^{l}<$ $\left(r_{1}^{l}-a_{12}^{u} M_{4}\right) / a_{11}^{u}$. Let us choose $m_{1}$ in such a way that

$$
0<\frac{r_{2}^{u}-\theta_{1}^{l}-\theta_{2}^{l}}{a_{21}^{l}}<m_{1}<\frac{r_{1}^{l}-a_{12}^{\mathrm{u}} M_{4}}{a_{11}^{u}} \Longrightarrow a_{21}^{l} m_{1}>r_{2}^{u}-\theta_{1}^{l}-\theta_{2}^{l}
$$

If $x\left(T_{3}\right) \geq m_{1}$, then $x(t) \geq m_{1}$, for all $t \geq T_{3}$. If $x\left(T_{3}\right)<m_{1}$ and let $\mu_{1}=x\left(T_{3}\right)\left(r_{1}^{l}-a_{11}^{u} m_{1}-\right.$ $\left.a_{12}^{u} M_{4}\right)>0$, then there exist an $\epsilon_{1}>0$, s.t. $x(t)<m_{1}$, and $\dot{x}_{1}(t)>\mu_{1}>0$, for all $t \in\left[T_{3}, T_{3}+\epsilon_{1}\right)$. Therefore, there exist a $T_{4}>T_{3}>0$, s.t. $x(t) \geq m_{1}$, for all $t \geq T_{4}$.

Define $V(t)=\min \left\{y_{1}(t), y_{2}(t), y_{3}(t)\right\}$. Then calculating the lower-right derivative of $V(t)$ along the solution of system (2.12) and (2.13), we obtainthe following.

(1) if $y_{1}(t) \leq y_{2}(t) \leq y_{3}(t)$, or, $y_{1}(t) \leq y_{3}(t) \leq y_{2}(t)$, then

$$
\begin{aligned}
D_{+} V(t) & =\dot{y}_{1}(t) \\
& \geq b_{1}^{l} y_{1}(t)-\theta_{1}^{u} y_{1}(t)-\delta_{1}^{u} y_{1}(t)-d^{u} C_{0}^{u} y_{1}(t)-\beta_{1}^{u} y_{1}^{2}(t) \\
& =\left(b_{1}^{l}-\theta_{1}^{u}-\delta_{1}^{u}-d^{u} C_{0}^{u}\right) y_{1}(t)-\beta_{1}^{u} y_{1}^{2}(t), \quad \forall t \geq 0 ;
\end{aligned}
$$

(2) if $y_{2}(t) \leq y_{1}(t) \leq y_{3}(t)$, or, $y_{2}(t) \leq y_{3}(t) \leq y_{1}(t)$, then

$$
\begin{aligned}
D_{+} V(t) & =\dot{y}_{2}(t) \\
& \geq b_{2}^{l} y_{2}(t)-\theta_{2}^{u} y_{2}(t)-\delta_{2}^{u} y_{2}(t)-\beta_{2}^{u} y_{2}^{2}(t) \\
& =\left(b_{2}^{l}-\theta_{2}^{u}-\delta_{2}^{u}\right) y_{2}(t)-\beta_{2}^{u} y_{2}^{2}(t), \quad \forall t \geq 0 ;
\end{aligned}
$$


(3) if $y_{3}(t) \leq y_{1}(t) \leq y_{2}(t)$, or, $y_{3}(t) \leq y_{2}(t) \leq y_{1}(t)$, then

$$
\begin{aligned}
D_{+} V(t) & =\dot{y}_{3}(t) \\
& \geq-r_{2}^{u} y_{3}(t)-a_{22}^{u} y_{3}^{2}(t)+a_{21}^{l} m_{1} y_{3}(t-\tau)+\left(\theta_{1}^{l}+\theta_{2}^{l}\right) y_{3}(t) \\
& =-\left(r_{2}^{u}-\theta_{1}^{l}-\theta_{2}^{l}\right) y_{3}(t)-a_{22}^{u} y_{3}^{2}(t)+a_{21}^{l} m_{1} y_{3}(t-\tau), \quad \forall t \geq T_{4}+\tau .
\end{aligned}
$$

Let us choose $m_{2}$ in such a way that $0<m_{2}<\min \left\{\left(b_{1}^{l}-\theta_{1}^{u}-\delta_{1}^{u}-d^{u} C_{0}^{u}\right) / \beta_{1}^{u},\left(b_{2}^{l}-\right.\right.$ $\left.\theta_{2}^{u}-\delta_{2}^{u}\right) / \beta_{2}^{u}$. From (3.12) and (3.13), we can obtain the followings:

(i) if $V(0)=\min \left\{y_{1}(0), y_{2}(0), y_{3}(0)\right\} \geq m_{2}$, then $\min \left\{y_{1}(t), y_{2}(t), y_{t}(t)\right\} \geq m_{2}$, for all $t \geq 0$;

(ii) if $V(0)=\min \left\{y_{1}(0), y_{2}(0), y_{3}(0)\right\}<m_{2}$, and let $\mu_{2}=\min \left[y_{1}(0)\left\{b_{1}^{l}-\theta_{1}^{u}-\delta_{1}^{u}-d^{u} C_{0}^{u}-\right.\right.$ $\left.\left.\beta_{1}^{u} m_{2}\right\}, y_{2}(0)\left\{b_{2}^{l}-\theta_{2}^{u}-\delta_{2}^{u}-\beta_{2}^{u} m_{2}\right\}\right]>0$, we consider the following two cases:

(a) $V(0)=y_{1}(0)<m_{2}$,

(b) $V(0)=y_{2}(0)<m_{2}$.

If (a) holds, then there exist an $\epsilon_{2}>0$, s.t. if $t \in\left[0, \epsilon_{2}\right), V(t)=y_{1}(t)<m_{2}$, and we have $D_{+} V(t)=\dot{y}_{1}(t)>\mu_{2}>0$.

If (b) holds, then there exist an $\epsilon_{3}>0$, s.t. if $t \in\left[0, \epsilon_{3}\right), V(t)=y_{2}(t)<m_{2}$, and we have $D_{+} V(t)=\dot{y}_{2}(t)>\mu_{2}>0$.

If (3.14) holds, then by (3.11) and Theorem 3.2, there exist $T_{5} \geq T_{4}+\tau>0$, s.t.

$$
y_{3}(t)>m_{3}, \quad \forall t \geq T_{5} \geq T_{4}+\tau>0, \text { where, } m_{3}<\frac{a_{21}^{l} m_{1}-\left(r_{2}^{u}-\theta_{1}^{l}-\theta_{2}^{l}\right)}{a_{22}^{u}} .
$$

From above discussions, we conclude that there exist $T_{6} \geq T_{5} \geq T_{4}+\tau>T_{3}>0$, s.t. $x(t) \geq m_{1}$ and $y_{i}(t) \geq m_{4} \quad(i=1,2,3)$, for all $t \geq T_{6}$ where

$$
\begin{gathered}
0<\frac{r_{2}^{u}-\theta_{1}^{l}-\theta_{2}^{l}}{a_{21}^{l}}<m_{1}<\frac{r_{1}^{l}-a_{12}^{u} M_{4}}{a_{11}^{u}}, \\
0<m_{4}<\min \left\{\frac{b_{1}^{l}-\theta_{1}^{u}-\delta_{1}^{u}-d^{u} C_{0}^{u}}{\beta_{1}^{u}}, \frac{b_{2}^{l}-\theta_{2}^{u}-\delta_{2}^{u}}{\beta_{2}^{u}}, \frac{a_{21}^{l} m_{1}-\left(r_{2}^{u}-\theta_{1}^{l}-\theta_{2}^{l}\right)}{a_{22}^{u}}\right\} .
\end{gathered}
$$

From the above discussions, we conclude that there exist $T_{6} \geq T_{5} \geq T_{4}+\tau>T_{3}>0$ s.t. every solution of system (2.12) and (2.13) eventually enters and remains in the region $\Omega=$ $\left\{\left(x, y_{1}, y_{2}, y_{3}\right) \mid m \leq x, y_{i} \leq M, i=1,2,3\right\}$, for all $t \geq T_{6}$, where $m=\min \left\{m_{1}, m_{4}\right\}$ and $M=\max \left\{M_{1}, M_{4}\right\}$, and $M_{1}$ is defined in Theorem 3.3. This completes the proof.

The results of Theorem 3.4 reveal the fact that lower values $\delta_{1}(t)$ (instantaneous per capita death rate function of nonprotective immature predator population), $\delta_{2}(t)$ 
(instantaneous per capita death rate function of protective immature predator population), $C_{0}(t)$ (concentration of toxicant in the immature predator organism in nonprotective patch at time $t), C_{e}(t)$ (concentration of toxicant in the environment at time $t$ ), and $d(t)$ (dose response parameter of nonprotective immature predator species to the organismal toxicant concentration at time $t$ ) lead to make the underlying system uniformly persistent.

\section{Global Asymptotic Stability of Periodic Solution}

From our everyday experience we know that the biological and environmental parameters are subject to fluctuation in time, and the effects of a periodically varying environment have an important selective forces on systems in a fluctuating environment. To investigate this kind of phenomenon, in the model, the coefficients should be periodic functions of time.

Suppose that system (2.12) is a periodic system with initial conditions (2.13). Here we assume that the periodic system (2.12) satisfies all conditions of Theorem 3.4. We derive sufficient conditions for all solutions of system (2.12) to converge to a periodic solution.

Theorem 4.1 (see [40]). Suppose that the continuous operator $A$ maps the closed and bounded convex set $Q \in R^{n}$ onto itself, then the operator $A$ has at least one fixed point in set $Q$.

Theorem 4.2. If periodic system (2.12) satisfies conditions of Theorem 3.4, then there is at least one strictly positive periodic solution of system (2.12)

Proof. From Theorems 3.3, 4.1 and 2 in Teng and Chen [41], it is easy to see that there is at least one strictly positive periodic solution of system (2.12).

Theorem 4.3. If system (2.12) has a periodic solution, then it is globally asymptotically stable provided lim inf $\operatorname{in}_{t \rightarrow \infty} A_{i}(t)>0,(i=1,2), \delta_{1}^{l}+d^{l} C_{0}^{l}+2 m \beta_{1}^{l}+D_{1}^{l}-D_{2}^{u}>0, \delta_{2}^{l}+2 m \beta_{2}^{l}+D_{2}^{l}-D_{1}^{u}>0$, where $A_{1}(t)=a_{11}^{l}-M a_{21}(t+\tau), A_{2}(t)=r_{2}^{l}+2 m a_{22}^{l}-a_{12}^{u}-b_{1}^{u}-b_{2}^{u}-M a_{21}(t+\tau), m, M$ are defined in Theorem 3.4.

Proof. Let $\left(u(t), v_{1}(t), v_{2}(t), v_{3}(t)\right)^{T}$ is a periodic solution of system (2.12) and (2.13). Suppose that $\left(x(t), y_{1}(t), y_{2}(t), y_{3}(t)\right)^{T}$ is any positive solution of (2.12) and (2.13). Let

$$
L_{1}(t)=|\ln x(t)-\ln u(t)|+\sum_{i=1}^{3}\left|y_{i}(t)-v_{i}(\mathrm{t})\right|
$$

Calculating the upper-right derivative of $L_{1}(t)$ along the positive solution of system (2.12) and (2.13), it follows that

$$
\begin{aligned}
D^{+} L_{1}(t)= & \left(\frac{\dot{x}(t)}{x(t)}-\frac{\dot{u}(t)}{u(t)}\right) \operatorname{sgn}(x(t)-u(t))+\sum_{i=1}^{3}\left(\dot{y}_{i}(t)-\dot{v}_{i}(t)\right) \operatorname{sgn}\left(y_{i}(t)-v_{i}(t)\right) \\
= & \operatorname{sgn}(x(t)-u(t))\left\{-a_{11}(t)(x(t)-u(t))-a_{12}(t)\left(y_{3}(t)-v_{3}(t)\right)\right\} \\
+ & \operatorname{sgn}\left(y_{1}(t)-v_{1}(t)\right)\left\{b_{1}(t)\left(y_{3}(t)-v_{3}(t)\right)-\left(\theta_{1}(t)+\delta_{1}(t)+d(t) C_{0}(t)\right)\left(y_{1}(t)-v_{1}(t)\right)\right. \\
& \left.-\beta_{1}(t)\left(y_{1}^{2}(t)-v_{1}^{2}(t)\right)+D_{1}(t)\left(\left(y_{2}(t)-v_{2}(t)\right)-\left(y_{1}(t)-v_{1}(t)\right)\right)\right\}
\end{aligned}
$$




$$
\begin{aligned}
&+\operatorname{sgn}\left(y_{2}(t)-v_{2}(t)\right)\{ b_{2}(t)\left(y_{3}(t)-v_{3}(t)\right)-\left(\theta_{2}(t)+\delta_{2}(t)\right)\left(y_{2}(t)-v_{2}(t)\right) \\
&\left.-\beta_{2}(t)\left(y_{2}^{2}(t)-v_{2}^{2}(t)\right)+D_{2}(t)\left(\left(y_{1}(t)-v_{1}(t)\right)-\left(y_{2}(t)-v_{2}(t)\right)\right)\right\} \\
&+\operatorname{sgn}\left(y_{3}(t)-v_{3}(t)\right)\{-r_{2}(t)\left(y_{3}(t)-v_{3}(t)\right)-a_{22}(t)\left(y_{3}^{2}(t)-v_{3}^{2}(t)\right)+a_{21}(t) y_{3}(t-\tau) \\
& \times(x(t-\tau)-u(t-\tau))+a_{21}(t) u(t-\tau)\left(y_{3}(t-\tau)-v_{3}(t-\tau)\right) \\
&\left.+\theta_{1}(t)\left(y_{1}(t)-v_{1}(t)\right)+\theta_{2}(t)\left(y_{2}(t)-v_{2}(t)\right)\right\} \\
& \leq-a_{11}^{l}|x(t)-u(t)|-\left(\delta_{1}^{l}+d^{l} C_{0}^{l}+2 m \beta_{1}^{l}+D_{1}^{l}-D_{2}^{u}\right)\left|y_{1}(t)-v_{1}(t)\right| \\
&-\left(\delta_{2}^{l}+2 m \beta_{2}^{l}+D_{2}^{l}-D_{1}^{u}\right)\left|y_{2}(t)-v_{2}(t)\right|-\left(r_{2}^{l}+2 m a_{22}^{l}-a_{12}^{u}-b_{1}^{u}-b_{2}^{u}\right)\left|y_{3}(t)-v_{3}(t)\right| \\
&+a_{21}(t) y_{3}(t-\tau)|x(t-\tau)-u(t-\tau)|+a_{21}(t) u(t-\tau)\left|y_{3}(t-\tau)-v_{3}(t-\tau)\right| .
\end{aligned}
$$

Let

$$
L_{2}(t)=\int_{t-\tau}^{t} a_{21}(s+\tau) y_{3}(s)|x(s)-u(s)| d s+\int_{t-\tau}^{t} a_{21}(s+\tau) u(s)\left|y_{3}(s)-v_{3}(s)\right| d s .
$$

Then,

$$
\begin{aligned}
D^{+} L_{2}(t)= & a_{21}(t+\tau) y_{3}(t)|x(t)-u(t)|-a_{21}(t) y_{3}(t-\tau)|x(t-\tau)-u(t-\tau)| \\
& +a_{21}(t+\tau) u(t)\left|y_{3}(t)-v_{3}(t)\right|-a_{21}(t) u(t-\tau)\left|y_{3}(t-\tau)-v_{3}(t-\tau)\right|
\end{aligned}
$$

Let

$$
L(t)=L_{1}(t)+L_{2}(t)
$$

Then,

$$
\begin{aligned}
D^{+} L(t) \leq & -\left(a_{11}^{l}-M a_{21}(t+\tau)\right)|x(t)-u(t)|-\left(\delta_{1}^{l}+d^{l} C_{0}^{l}+2 m \beta_{1}^{l}+D_{1}^{l}-D_{2}^{u}\right)\left|y_{1}(t)-v_{1}(t)\right| \\
& -\left(\delta_{2}^{l}+2 m \beta_{2}^{l}+D_{2}^{l}-D_{1}^{u}\right)\left|y_{2}(t)-v_{2}(t)\right| \\
& -\left(r_{2}^{l}+2 m a_{22}^{l}-a_{12}^{u}-b_{1}^{u}-b_{2}^{u}-M a_{21}(t+\tau)\right)\left|y_{3}(t)-v_{3}(t)\right| \\
= & -A_{1}(t)|x(t)-u(t)|-\left(\delta_{1}^{l}+d^{l} C_{0}^{l}+2 m \beta_{1}^{l}+D_{1}^{l}-D_{2}^{u}\right)\left|y_{1}(t)-v_{1}(t)\right| \\
& -\left(\delta_{2}^{l}+2 m \beta_{2}^{l}+D_{2}^{l}-D_{1}^{u}\right)\left|y_{2}(t)-v_{2}(t)\right|-A_{2}(t)\left|y_{3}(t)-v_{3}(t)\right|,
\end{aligned}
$$


where $A_{1}(t)=a_{11}^{l}-M a_{21}(t+\tau), A_{2}(t)=r_{2}^{l}+2 m a_{22}^{l}-a_{12}^{u}-b_{1}^{u}-b_{2}^{u}-M a_{21}(t+\tau)$. By assumption, $\delta_{1}^{l}+d^{l} C_{0}^{l}+2 m \beta_{1}^{l}+D_{1}^{l}-D_{2}^{u}>0, \delta_{2}^{l}+2 m \beta_{2}^{l}+D_{2}^{l}-D_{1}^{u}>0$, and there exist $\alpha_{1}>0, \alpha_{2}>0, T^{*} \geq T_{6}>0$, ( $T_{6}$ is defined in Theorem 3.4), such that $A_{1}(t) \geq \alpha_{1}, A_{2}(t) \geq \alpha_{2}$, for all $t \geq T^{*}$. Integrating both sides of (4.6) on the interval $\left[T^{*}, t\right]$, we get

$$
\begin{gathered}
L(t)+\int_{T^{*}}^{t} A_{1}(s)|x(s)-u(s)| d s+\left(\delta_{1}^{l}+d^{l} C_{0}^{l}+2 m \beta_{1}^{l}+D_{1}^{l}-D_{2}^{u}\right) \int_{T^{*}}^{t}\left|y_{1}(s)-v_{1}(s)\right| d s \\
+\left(\delta_{2}^{l}+2 m \beta_{2}^{l}+D_{2}^{l}-D_{1}^{u}\right) \int_{T^{*}}^{t}\left|y_{2}(s)-v_{2}(s)\right| d s+\int_{T^{*}}^{t} A_{2}(s)\left|y_{3}(s)-v_{3}(s)\right| d s \leq L\left(T^{*}\right) \\
\Longrightarrow L(t)+\alpha_{1} \int_{T^{*}}^{t}|x(s)-u(s)| d s+\left(\delta_{1}^{l}+d^{l} C_{0}^{l}+2 m \beta_{1}^{l}+D_{1}^{l}-D_{2}^{u}\right) \int_{T^{*}}^{t}\left|y_{1}(s)-v_{1}(s)\right| d s \\
+\left(\delta_{2}^{l}+2 m \beta_{2}^{l}+D_{2}^{l}-D_{1}^{u}\right) \int_{T^{*}}^{t}\left|y_{2}(s)-v_{2}(s)\right| d s+\alpha_{2} \int_{T^{*}}^{t}\left|y_{3}(s)-v_{3}(s)\right| d s \leq L\left(T^{*}\right) \\
\Longrightarrow L(t) \text { is bounded on }\left[T^{*}, \infty\right) \text { and also } \int_{T^{*}}^{\infty}|x(s)-u(s)| d s<\infty \\
\int_{T^{*}}^{\infty}\left|y_{i}(s)-v_{i}(s)\right| d s<\infty \quad(i=1,2,3) .
\end{gathered}
$$

By Theorem 3.3, $|x(s)-u(s)|,\left|y_{i}(s)-v_{i}(s)\right|(i=1,2,3)$ are bounded on $\left[T^{*}, \infty\right)$. On the other hand, it is easy to see that $\dot{x}(t), \dot{u}(t), \dot{y}_{i}(t)$, and $\dot{v}_{i}(t) \quad(i=1,2,3)$ are bounded for $t \geq T^{*}$. Therefore, $|x(t)-u(t)|,\left|y_{i}(t)-v_{i}(t)\right|(i=1,2,3)$ are uniformly continuous on $\left[T^{*}, \infty\right)$. By Barbalat's Lemma [42], we conclude that

$$
|x(t)-u(t)| \longrightarrow 0, \quad\left|y_{i}(t)-v_{i}(t)\right| \longrightarrow 0 \quad \text { as } t \longrightarrow \infty, i=1,2,3
$$

This completes the proof.

From Theorems 3.4 and 4.3 we observe that the diffusion coefficient functions and time delay have no effect on the uniformly persistent of the system (2.12) but they have some effect on the global asymptotic stability of this system.

\section{Conclusions}

In this paper, we have considered pollution in a nonautonomous Lotka-Volterra type predator-prey system together with diffusional migration among the immature predator population between protective and nonprotective patches. A constant delay among the matured predators due to the gestation of the predators has been incorporated. It is also assumed that the mature adult predators can only contribute to the reproduction of the predator biomass and that the change rate of predators depends on the number of the preys and of the predators presented at some previous time. Here we incorporate a stage-structure for the predators and assume that the toxicants have no effect on the mature species. 
The toxic of polluted population comes from the environment. Suppose that environment of patch 1 is polluted nonprotective region, and patch 2 is the protective region for the immature predator population which is only affected by pollution. In order to protect the existence of the polluted population, we can use the artificial method, the one's own purification function of the population, toxin in the body of the individuals in nonprotective patch can be removed, and then we will bring them into protective patch. The scale of the protective zone can be regulated through diffusive coefficients $D_{i}(t), i=1,2$.

We have obtained the survival threshold of each population of this system. Our mathematical results reveal the fact that lower values $\delta_{1}(t)$ (instantaneous per capita death rate function of nonprotective immature predator population), $\delta_{2}(t)$ (instantaneous per capita death rate function of protective immature predator population), $C_{0}(t)$ (concentration of toxicant in the immature predator organism in nonprotective patch at time $t), C_{e}(t)$ (concentration of toxicant in the environment at time $t$ ), and $d(t)$ (dose response parameter of nonprotective immature predator species to the organismal toxicant concentration at time $t$ ) lead to make the underlying system uniformly persistent. By constructing an appropriate Lyapunov function, we have derived sufficient conditions to confirm that if this system admits a positive periodic solution, then it is globally asymptotically stable. We have observed that the diffusion coefficient functions and time delay have no effect on the uniformly persistent of the system (2.12) but they have some effect on the global asymptotic stability of this system.

In future work, it would be interesting to expand on simulations by using some current realistic data to estimate the parameters. The collection of data relevant to key parameters by ecologists and environmentalists at a global level would be of significant use from an implementation and policy perspective.

\section{Acknowledgments}

The author is very grateful to the anonymous referees and the Editor (Dr. Harvinder S. Sidhu) for their careful reading, valuable comments, and helpful suggestions, which have helped him to improve the presentation of this work significantly. He is grateful to Professor A.Dvurecenskij, Dr. Vrto, Mathematical Institute, Slovak Academy of Sciences, Bratislava, Slovak Republic, and Dr. D. N. Garain, P. G. Department of Mathematics, S. K. M. University, Dumka, Jharkhand, India for their helps and encouragements. He is thankful to Slovak Academic Information Agency (SAIA), Bratislava, Slovak Republic for financial support.

\section{References}

[1] G. E. Hutchinson, A Treatise on Limnology, vol. 1, John Wiley \& Sons, New York, NY, USA, 1957.

[2] E. C. Tsivoglou, Tracer Measurement of Stream Reaeration, Water Pollution Control Administration, Washington, DC, USA, 1970.

[3] G. E. Russell-Hunter, Aquatic Productivity, Collier Macmillan, Toronto, Canada, 1970.

[4] N. L. Miller, "Stability analysis of toxic substances within aquatic ecosystems and their effect on aquatic populations," Ecological Modelling, vol. 60, no. 2, pp. 151-165, 1992.

[5] J. R. E. Jones, Fish and River Pollution, Butterworths, Washington, DC, USA, 1964.

[6] G. Howells, Acid Rain and Acid Water, Ellis Horwood, Chichester, UK, 1990.

[7] S. A. Nelson, "The problem of oil pollution of the sea," in Advances in Marine Biology, Academic Press, London, UK, 1970. 
[8] J. B. Shukla, H. I. Freedman, V. N. Pal, O. P. Misra, M. Agarwal, and A. Shukla, "Degradation and subsequent regeneration of a forestry resource: a mathematical model," Ecological Modelling, vol. 44, no. 3-4, pp. 219-229, 1989.

[9] J. N. Woodman and E. B. Cowling, "Airborne chemicals and forest health: convincing data are lacking to prove the case for injury caused by atmospheric pollutants other than ozone," Environmental Science and Technology, vol. 21, no. 2, pp. 120-126, 1987.

[10] C. N. Haas, "Application of predator-prey models to disinfection," Journal of the Water Pollution Control Federation, vol. 53, no. 3I, pp. 378-386, 1981.

[11] A. L. Jensen and J. S. Marshall, "Application of a surplus production model to assess environmental impacts on exploited populations of Daphnia pulex in the laboratory," Environmental Pollution Series A, vol. 28, no. 4, pp. 273-280, 1982.

[12] T. G. Hallam and C. E. Clark, "Nonautonomous logistic equations as models of populations in a deteriorating environment," Journal of Theoretical Biology, vol. 93, no. 2, pp. 303-311, 1981.

[13] T. G. Hallam, C. E. Clark, and G. S. Jordan, "Effects of toxicants on populations: a qualitative approach. II. First order kinetics," Journal of Mathematical Biology, vol. 18, no. 1, pp. 25-37, 1983.

[14] T. G. Hallam, C. E. Clark, and R. R. Lassiter, "Effects of toxicants on populations: a qualitative approach. I. Equilibrium environmental exposure," Ecological Modelling, vol. 18, no. 4, pp. 291-304, 1983.

[15] T. G. Hallam and J. T. De Luna, "Effects of toxicants on populations: a qualitative approach. III. Environmental and food chain pathways," Journal of Theoretical Biology, vol. 109, no. 3, pp. 411-429, 1984.

[16] J. T. De Luna and T. G. Hallam, "Effects of toxicants on populations: a qualitative approach IV. Resource-consumer-toxicant models," Ecological Modelling, vol. 35, no. 3-4, pp. 249-273, 1987.

[17] M. Zhien, C. Guirong, and W. Wendi, "Persistence and extinction of a population in a polluted environment," Mathematical Biosciences, vol. 101, no. 1, pp. 75-97, 1990.

[18] L. Huaping and M. Zhien, "The threshold of survival for system of two species in a polluted environment," Journal of Mathematical Biology, vol. 30, no. 1, pp. 49-61, 1991.

[19] H. I. Freedman and J. B. Shukla, "Models for the effect of toxicant in single-species and predator-prey systems," Journal of Mathematical Biology, vol. 30, no. 1, pp. 15-30, 1991.

[20] W. D. Wang and Z. E. Ma, "Permanence of populations in a polluted environment," Mathematical Biosciences, vol. 122, no. 2, pp. 235-248, 1994.

[21] B. Dubey, "Modelling the effect of toxicant on forestry resources," Indian Journal of Pure and Applied Mathematics, vol. 28, no. 1, pp. 1-12, 1997.

[22] Y. Xiao and L. Chen, "Effects of toxicants on a stage-structured population growth model," Applied Mathematics and Computation, vol. 123, no. 1, pp. 63-73, 2001.

[23] M. Ghosh, P. Chandra, and P. Sinha, "A mathematical model to study the effect of toxic chemicals on a prey-predator type fishery," Journal of Biological Systems, vol. 10, no. 2, pp. 97-105, 2002.

[24] B. Buonomo and D. Lacitignola, "General conditions for global stability in a single species populationtoxicant model," Nonlinear Analysis: Real World Applications, vol. 5, no. 4, pp. 749-762, 2004.

[25] Z. Li, Z. Shuai, and K. Wang, "Persistence and extinction of single population in a polluted environment," Electronic Journal of Differential Equations, no. 108, pp. 1-5, 2004.

[26] G. P. Samanta and A. Maiti, "Dynamical model of a single-species system in a polluted environment," Journal of Applied Mathematics E Computing, vol. 16, no. 1-2, pp. 231-242, 2004.

[27] J. Wang and K. Wang, "Analysis of a single species with diffusion in a polluted environment," Electronic Journal of Differential Equations, no. 112, pp. 1-11, 2006.

[28] J. He and K. Wang, "The survival analysis for a single-species population model in a polluted environment," Applied Mathematical Modelling, vol. 31, no. 10, pp. 2227-2238, 2007.

[29] J. He and K. Wang, "On population in a polluted patchy environment," Journal of Physics: Conference Series, vol. 96, no. 1, pp. 1-5, 2008.

[30] T. Das, R. N. Mukherjee, and K. S. Chaudhuri, "Harvesting of a prey-predator fishery in the presence of toxicity," Applied Mathematical Modelling, vol. 33, no. 5, pp. 2282-2292, 2009.

[31] R. Xu and Z. Ma, "The effect of stage-structure on the permanence of a predator-prey system with time delay," Applied Mathematics and Computation, vol. 189, no. 2, pp. 1164-1177, 2007.

[32] S. Gao, L. Chen, and Z. Teng, "Hopf bifurcation and global stability for a delayed predator-prey system with stage structure for predator," Applied Mathematics and Computation, vol. 202, no. 2, pp. 721-729, 2008. 
[33] J. Wang, Q. Lu, and Z. Feng, "A nonautonomous predator-prey system with stage structure and double time delays," Journal of Computational and Applied Mathematics, vol. 230, no. 1, pp. 283-299, 2009.

[34] X. Zhou, X. Shi, and X. Song, "Analysis of nonautonomous predator-prey model with nonlinear diffusion and time delay," Applied Mathematics and Computation, vol. 196, no. 1, pp. 129-136, 2008.

[35] W. J. Manning and W. A. Feder, Biomonitoring Air Pollutants with Plants, Applied Science, Barking, UK, 1980.

[36] T. G. Hallam and Z. E. Ma, "Persistence in population models with demographic fluctuations," Journal of Mathematical Biology, vol. 24, no. 3, pp. 327-339, 1986.

[37] Z. E. Ma and T. G. Hallam, "Effects of parameter fluctuations on community survival," Mathematical Biosciences, vol. 86, no. 1, pp. 35-49, 1987.

[38] J. Hale, Theory of Functional Differential Equations, Applied Mathematical Sciences, Springer, New York, NY, USA, 2nd edition, 1977.

[39] X. Song and L. Chen, "Optimal harvesting and stability for a two-species competitive system with stage structure," Mathematical Biosciences, vol. 170, no. 2, pp. 173-186, 2001.

[40] M. W. Hirsch, Differential Topology, Springer, New York, NY, USA, 1980.

[41] Z. D. Teng and L. S. Chen, "Positive periodic solutions of periodic Kolmogorov type systems with delays," Acta Mathematicae Applicatae Sinica, vol. 22, no. 3, pp. 446-456, 1999 (Chinese).

[42] K. Gopalsamy, Stability and Oscillations in Delay Differential Equations of Population Dynamics, vol. 74 of Mathematics and Its Applications, Kluwer Academic Publishers, Dordrecht, The Netherlands, 1992. 


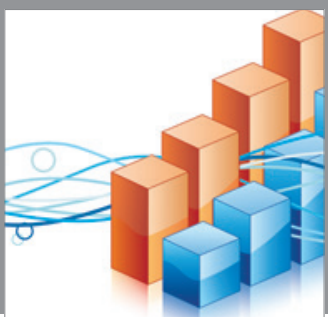

Advances in

Operations Research

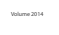

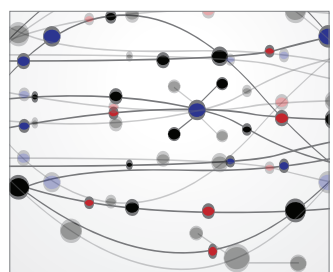

\section{The Scientific} World Journal
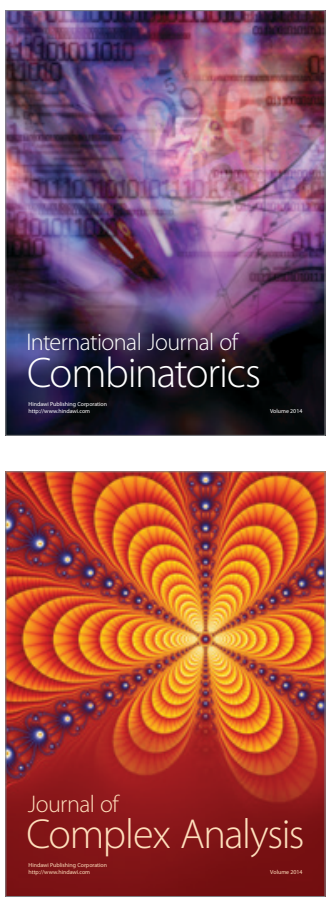

International Journal of

Mathematics and

Mathematical

Sciences
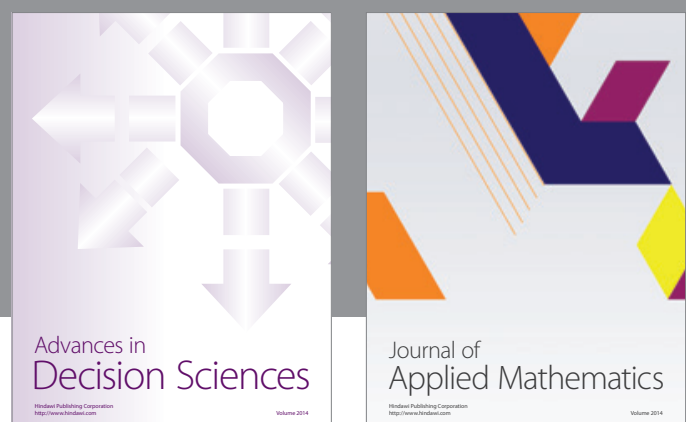

Journal of

Applied Mathematics
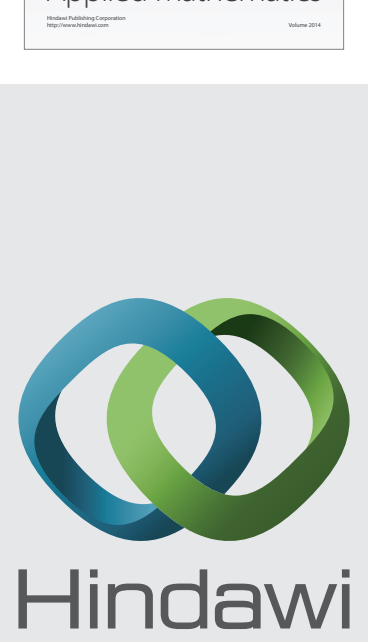

Submit your manuscripts at http://www.hindawi.com
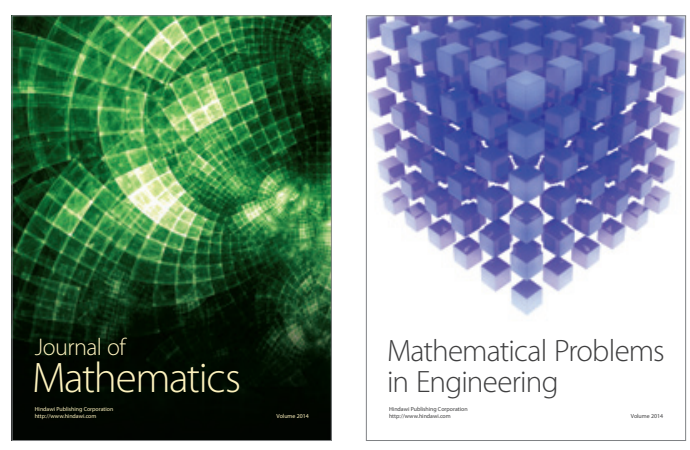

Mathematical Problems in Engineering
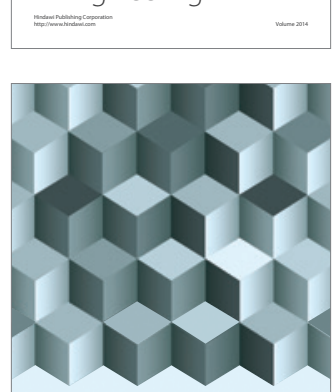

Journal of

Function Spaces
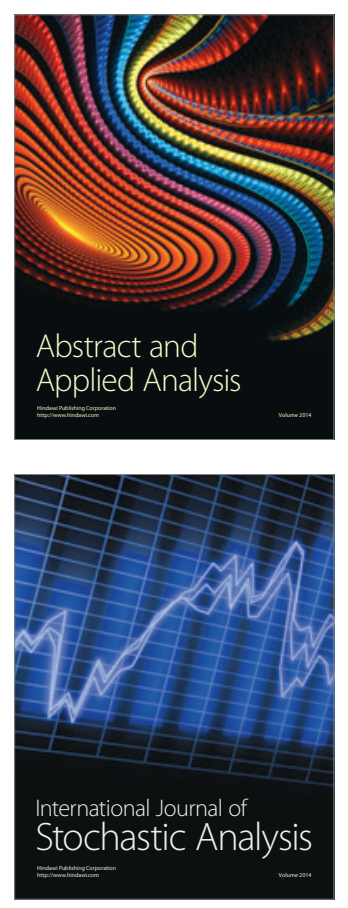

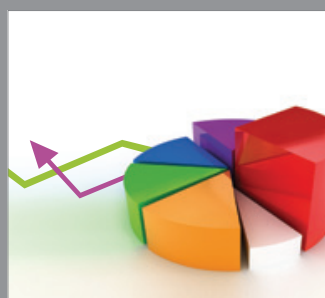

ournal of

Probability and Statistics

Promensencen
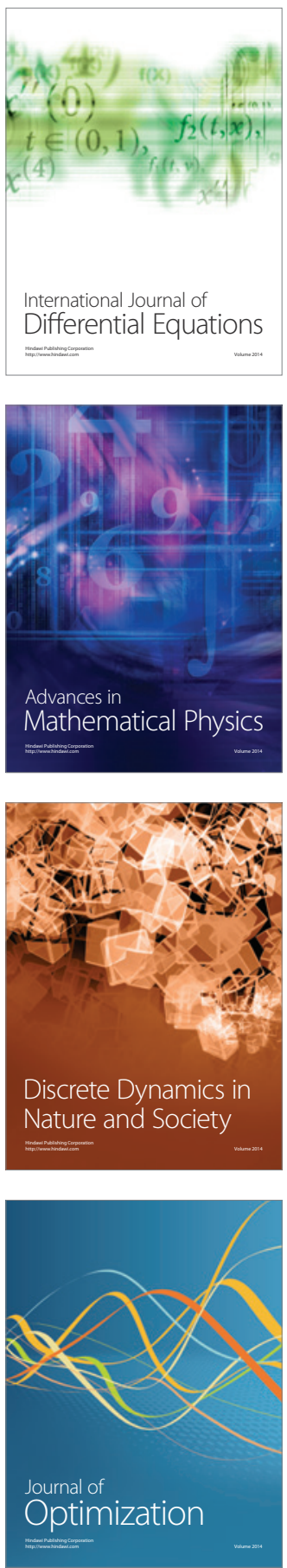